\title{
Increased levels of interleukin-8 in BAL fluid from smokers susceptible to pulmonary emphysema
}

\author{
M Tanino, T Betsuyaku, K Takeyabu, Y Tanino, E Yamaguchi, K Miyamoto, M \\ Nishimura
}

Thorax 2002;57:405-411

See end of article for authors' affiliations

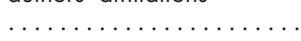

Correspondence to: Dr M Tanino, First Department of Medicine, Hokkaido University School of Medicine, North 15 West 7, Kita-ku, Sapporo, 060-8638, Japan; tanino@med.hokudai.ac.jp

Revised version received 30 October 2001 Accepted for publication 19 November 2001

\begin{abstract}
Background: It has previously been shown that smokers with computed tomographic (CT) evidence of subclinical emphysema have signs of neutrophil activation, despite having no appreciable increase in the number of neutrophils in their bronchoalveolar lavage (BAL) fluid.

Methods: The levels of the following chemoattractants in BAL fluid from 61 community based older volunteers classified into four groups according to current smoking status and the presence or absence of emphysema were determined: interleukin 8 (IL-8), epithelial neutrophil activating protein 78 (ENA-78) and leukotriene $B_{4}\left(L_{T} B_{4}\right)$ which are primarily chemotactic for neutrophils; monocyte chemoattractant protein 1 (MCP-1) and macrophage inflammatory protein-1 $\alpha$ (MIP-1 $\alpha)$ which are predominantly chemotactic for mononuclear leucocytes.

Results: Of the five chemoattractants studied, only the level of IL-8 in BAL fluid clearly distinguished between subjects with and without emphysema among current smokers (median values 34.7 and $12.2 \mathrm{pg} / \mathrm{ml}$, respectively, $\mathrm{p}<0.01)$. In addition, the levels of IL-8 and neutrophil elastase- $\alpha$, protease inhibitor complex in BAL fluid were significantly correlated $(r=0.65, p<0.01)$. There was no difference in either the release of IL-8 from cultured alveolar macrophages at 24 hours or the expression of IL-8 messenger RNA of alveolar macrophages in the two groups of current smokers with and without emphysema.

Conclusion: An accelerated response of IL-8 to chronic smoking is a factor that characterises those smokers who are susceptible to pulmonary emphysema, although the cellular source of IL-8 remains to be determined.
\end{abstract}

$\mathrm{T}$ he pathophysiology of pulmonary emphysema has been characterised by a proteinase-antiproteinase imbalance, followed by the progressive destruction of elastin and other extracellular components. ${ }^{1}$ Smoking is a well known exogenous cause of emphysema and is believed to recruit inflammatory cells such as neutrophils and/or macrophages which contain a variety of proteolytic enzymes. However, it is still not certain which cell mainly contributes to the pathogenesis of pulmonary emphysema and it also unknown why only a small proportion of smokers develop clinically apparent emphysema.

We have tried to identify the factors that distinguish subjects who are asymptomatic but who are diagnosed as having pulmonary emphysema (subclinical emphysema) by high resolution computed tomographic (HRCT) scans from age matched controls with a similar smoking history who do not have any signs of emphysema. We have found that the level of neutrophil elastase- $\alpha_{1}$ protease inhibitor complex $\left(\mathrm{NE}-\alpha_{1} \mathrm{PI}\right)$ is significantly increased in the bronchoalveolar lavage (BAL) fluid of patients with subclinical emphysema, ${ }^{2}$ and the level of this complex is positively correlated with that of elastin-derived peptides (EDP) in BAL fluid. ${ }^{3}$ We have also shown that the amounts of degranulated proteins of neutrophils such as human neutrophil lipocalin (HNL), neutrophil collagenase (MMP-8), and gelatinase B (MMP-9) are also significantly higher in the BAL fluid from these subjects. ${ }^{4}$ Although these results support the involvement of neutrophils in the early development of pulmonary emphysema, there is no appreciable increase in the number of neutrophils in the BAL fluid of subjects with pulmonary emphysema compared with smoking controls without pulmonary emphysema. The surprising dissociation of increased markers of neutrophil accumulation and/or activation from the number of neutrophils in the BAL fluid of these subjects prompted us to examine the role of chemotactic signals in the alveolar space.

We first measured the levels of three chemoattractants for neutrophils in BAL fluid-interleukin 8 (IL-8), ${ }^{5}$ epithelial neutrophil activating protein 78 (ENA-78), ${ }^{6}$ and leukotriene $\mathrm{B}_{4}\left(\mathrm{LTB}_{4}\right)^{7}$-and, for comparison, the levels of monocyte chemoattractant protein 1 (MCP-1) and macrophage inflammatory protein $1 \alpha(\mathrm{MIP}-1 \alpha)$, both of which are predominantly chemotactic for mononuclear leucocytes. ${ }^{5}$ Since IL-8 was found to distinguish between current smokers with and without emphysema, we then tested the hypothesis that alveolar macrophages (AMs) are one of the sources of IL-8 that contribute to the early development of pulmonary emphysema.

\section{METHODS}

\section{Study population}

Sixty one community based asymptomatic subjects were recruited from our smoking cessation clinics, non-pulmonary clinics, and municipal community centres: 16 former smokers who had ceased smoking for a minimum of 6 months (15 men/one woman, mean (SE) age 61 (3) years) and 45 current smokers (40 men/five women, mean (SE) age 53 (2) years). After informed consent was obtained, they were first screened for pulmonary emphysema by HRCT scanning (Yokokawa CT

Abbreviations: ENA-78, epithelial neutrophil activating protein 78; $\mathrm{FEV}_{1}$, forced expiratory volume in 1 second; FVC, forced vital capacity; IL-8, interleukin 8; $\mathrm{LTB}_{4}$, leukotriene $\mathrm{B}_{4} ; \mathrm{MCP}-1$, monocyte chemoattractant protein $1 ;$ MIP-1 $\alpha$, macrophage inflammatory protein $1 \alpha ; \mathrm{NE}-\alpha_{1} \mathrm{PI}$, neutrophil elastase- $\alpha_{1}$ protease inhibitor complex; TLCO, carbon monoxide transfer factor; VC, vital capacity. 
Table 1 Clinical characteristics and pulmonary function data

\begin{tabular}{|c|c|c|c|c|}
\hline & \multicolumn{2}{|l|}{ Former smokers } & \multicolumn{2}{|l|}{ Current smokers } \\
\hline & Emphysema (-) & Emphysema (+) & Emphysema (-) & Emphysema (+) \\
\hline Number of subjects & 7 & 9 & 20 & 25 \\
\hline Age (years) & $64(3)$ & $59(4)$ & $51(2)^{*}$ & $54(2)^{*}$ \\
\hline Pack years of smoking & $20(8)$ & $48(7)^{*}$ & $44(3)^{*}$ & $46(5)^{*}$ \\
\hline VC (\% pred) & $129.2(2.1)$ & $105.0(2.5)^{*}$ & $110.3(3.2)^{*}$ & $105.7(3.2)^{*}$ \\
\hline $\mathrm{FEV}_{1}(\%$ pred) & $124.1(8.9)$ & $87.9(5.7)^{*}$ & $101.9(3.5)^{*} \dagger$ & $89.2(3.3)^{*} \ddagger$ \\
\hline $\mathrm{FEV}_{1} / \mathrm{FVC}(\%)$ & $78.9(1.3)$ & $71.6(3.0)$ & $80.0(1.4) \dagger$ & $71.6(2.1) \ddagger$ \\
\hline TLCO (\% pred) & $123.1(10.0)$ & $87.3(5.1)^{*}$ & $99.0(3.1)^{*}$ & $85.7(3.3)^{*} \ddagger$ \\
\hline
\end{tabular}

Values are mean (SE)

${ }^{*} p<0.05 v$ former smokers without emphysema; $\dagger p<0.05 v$ former smokers with emphysema; $\ddagger p<0.05 v$ current smokers without emphysema.

\begin{tabular}{|c|c|c|c|c|}
\hline & \multicolumn{2}{|l|}{ Former smokers } & \multicolumn{2}{|l|}{ Current smokers } \\
\hline & Emphysema (-) & Emphysema (+) & Emphysema (-) & Emphysema (+) \\
\hline Recovery (\%) & $56.0(5.0)$ & $37.6(5.6)^{*} \ddagger$ & $50.5(3.1)$ & $41.7(3.4)^{*}$ \\
\hline Albumin (mg/l) & 54.5 (12.3) & $53.3(11.9)^{\top}$ & $34.9(4.4)$ & $54.7(9.8)$ \\
\hline Total cells $\left(\times 10^{4} / \mathrm{ml} \mathrm{BAL}\right.$ fluid) & $8.5(1.7)$ & $12.2(2.3)$ & $35.4(6.8)$ & $32.1(6.7)$ \\
\hline Viability (\%) & $87.1(1.3)$ & $85.8(3.2)$ & $92.5(1.9)$ & $87.1(2.2)$ \\
\hline $\begin{array}{l}\text { Macrophages }\left(\times 10^{4} / \mathrm{ml} \mathrm{BAL}\right. \\
\text { fluid) }\end{array}$ & $7.3(1.4)$ & $9.2(1.5)$ & $34.5(6.8)^{*} \dagger$ & $31.3(6.7) * \dagger$ \\
\hline Neutrophils $\left(\times 10^{4} / \mathrm{ml} \mathrm{BAL}\right.$ fluid) & $0.03(0.01)$ & $0.20(0.09)$ & $0.31(0.10)$ & $0.36(0.16)$ \\
\hline
\end{tabular}

9000 unit, Yokokawa; or Siemens Somatom PLVS 40, Siemens; or Toshiba TCT X-Vigor, Toshiba). Serial horizontal slices of 1-2 mm width were obtained at $10 \mathrm{~mm}$ intervals from the apex to below the diaphragm. The HRCT scans set at a window level of -700 to -800 Hounsfield units (HU) and a width of 1000-1500 HU were evaluated by three pulmonary physicians, who were blind to any individual information, for the presence of emphysema in all serial horizontal slices. Subjects were considered to have emphysema regardless of size or severity based on the agreement of all three doctors. None of the subjects was on regular medication nor had a history of asthma. None had symptoms of a cold, nor had any taken any medication for 2 months before the study. To confirm the current smoking status objectively, 42 subjects were examined for exhaled CO levels using a CO analyser (Micro Smokerlyser, Bedfont, Kent, UK), and the serum cotinine levels of 19 subjects were also examined. ${ }^{2}$ Following the HRCT scans, subjects underwent pulmonary function tests and blood sampling. After elimination of non-eligible subjects, the remaining subjects were divided into four groups according to current smoking status and the existence of emphysematous changes.

The study was approved by the ethics committee of Hokkaido University School of Medicine.

\section{Bronchoalveolar lavage (BAL)}

The smokers were asked to refrain from smoking for at least 12 hours before the BAL procedure to remove the acute effect of smoking. Sequential BAL was performed as previously described. ${ }^{2}$ In brief, four separate $50 \mathrm{ml}$ aliquots of sterile $0.9 \%$ saline were instilled into the right middle lobe segment of the lungs through a wedged flexible fibreoptic bronchoscope (Olympus BF-B3R, Tokyo, Japan) and then gently suctioned. The fluid returned from the first $50 \mathrm{ml}$ aliquot was not used because fluid from the first aliquot is known to contain cells and material that comes mostly from the large airways rather than the peripheral regions. The remaining lavage fluid was combined and used as the alveolar lavage fluid rich fraction for this study.

\section{Processing of BAL fluid}

Recovered BAL fluid and cell analysis were carried out for each individual as previously described. ${ }^{2}$ Cell free BAL fluid was concentrated with Centriprep concentrators (Amicon, Beverly, MA, USA) having a molecular weight cut off of $3 \mathrm{kD}$ for analysis of IL-8, MCP-1, and MIP- $1 \alpha$.

\section{Culture of alveolar macrophages}

AMs were isolated by adherence onto poly-L-lysine coated plastic wells (Corning, NY, USA) for 1 hour at $37^{\circ} \mathrm{C}$ in RPMI-1640 medium (Gibco, Grand Island, NY, USA) containing 100 U/ml penicillin and $100 \mathrm{mg} / \mathrm{ml}$ streptomycin in a moist atmosphere of 95\% air and 5\% $\mathrm{CO}_{2}$. After removal of non-adherent cells with two phosphate buffered saline (PBS) washes, the remaining cells were cultured in RPMI-1640 containing antibiotics for an additional 24 hours as previously described. ${ }^{8}$

\section{Analysis of IL-8}

The levels of IL-8 in concentrated BAL fluid and culture medium of AMs were quantified using a Quantikine IL-8 enzyme linked immunoassay (ELISA) kit (RED Systems, MN, USA) according to the protocol supplied with the kit. A standard curve was obtained with serial dilution of the supplied recombinant human IL-8 by linear regression (minimal detection limit 6 pg/ $\mathrm{ml}$ ). The concentration of IL-8 in each sample was obtained by interpolation of its absorbance from a standard curve, and the mean value of the duplicate samples was then taken as the representative value. The correlation coefficient of the curve of the supplied IL-8 standards was $>0.90$. Levels of IL-8 in BAL fluid were expressed per $\mathrm{ml}$ of unconcentrated BAL fluid by adjusting for the degree to which each BAL fluid sample was concentrated. The concentration of IL-8 culture medium of AMs was adjusted to $1 \times 10^{6}$ macrophages.

\section{Enzyme immunoassay for ENA-78 and LTB $_{4}$}

Analysis of ENA-78 in unconcentrated BAL fluid was performed with a human ENA-78 immunoassay kit (R\&D Systems) with a detection limit of $15 \mathrm{pg} / \mathrm{ml}$. Analysis of $\mathrm{LTB}_{4}$ 


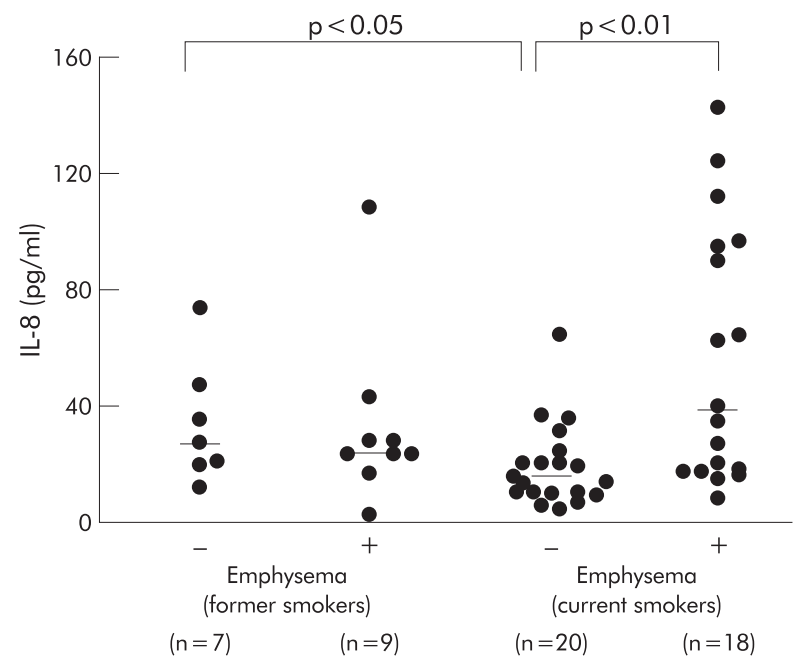

Figure 1 Level of IL-8 in BAL fluid. The level was significantly increased in current smokers with emphysema and former smokers without emphysema compared with current smokers without emphysema. Median values are shown as horizontal lines.

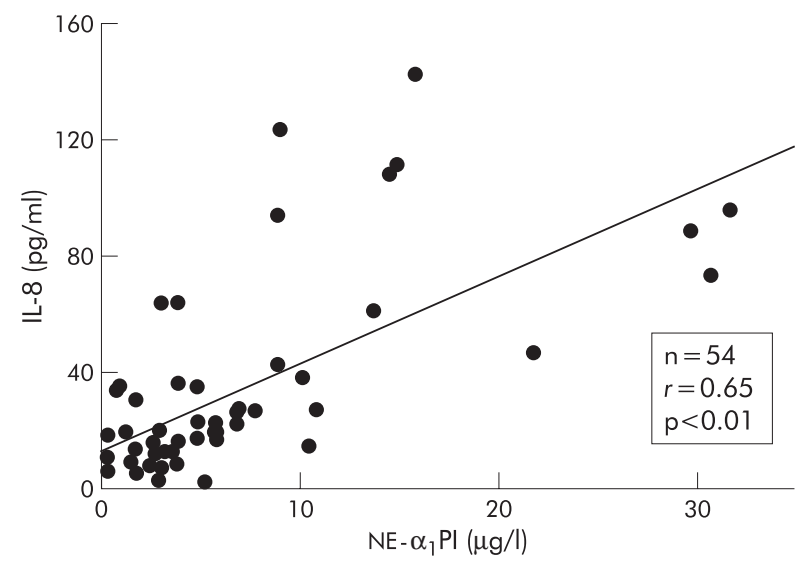

Figure 2 Relationship between IL-8 and NE- $\alpha_{1}$ PI in BAL fluid. Each point represents the data from individual samples. Correlations were conducted with Spearman rank correlation test.

in unconcentrated BAL fluid was carried out using an $\mathrm{LTB}_{4}$ enzyme immunoassay (EIA) kit (Amersham Life Science, UK) with a detection limit of $6 \mathrm{pg} / \mathrm{ml}$; cross reactivity with $\mathrm{LTC}_{4^{\prime}}$ LTD $_{4}$, LTE $_{4}$, 5-hydroxyeicosatetraenoic acid (5-HETE), 12HETE, and 15-HETE was less than $0.04 \%$.

\section{NE- $\alpha_{1}$ PI quantification}

Measurement of NE- $\alpha_{1}$ PI in unconcentrated BAL fluid was as previously reported. ${ }^{2}$

\section{Quantification of MCP-1 and MIP- $1 \alpha$}

Quantitative analysis of MCP-1 and MIP- $1 \alpha$ in concentrated BAL fluid was performed using EIA kits for MCP-1 (Hycult Biotechnology, Netherlands) and MIP-l $\alpha$ (R\&D Systems) with detection limits of $6 \mathrm{pg} / \mathrm{ml}$ and $8 \mathrm{pg} / \mathrm{ml}$, respectively; there was no cross reactivity with other cytokines tested by the manufacturer.

\section{Quantitative RT-PCR for IL-8}

Total RNA was prepared from AMs by the guanidinium thiocyanate phenol chloroform method using the RNA extraction kit Isogen (Nippon Gene, Tokyo, Japan). Single strand complementary DNA (cDNA) for the PCR template was synthesised using Molony murine leukemia virus (MMLV)

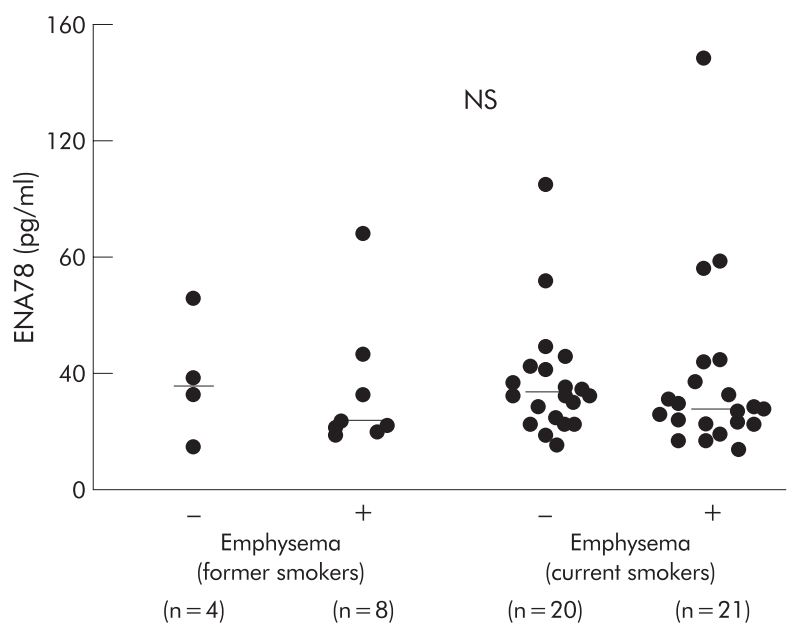

Figure 3 Level of ENA-78 in BAL fluid. There was no significant difference between the four groups. Median values are shown as horizontal lines.

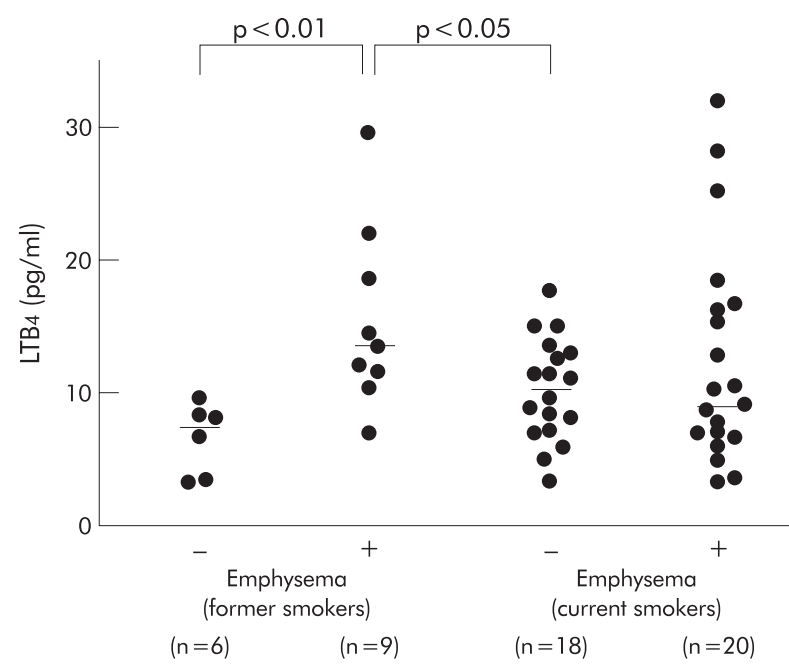

Figure 4 Level of $\mathrm{LTB}_{4}$ in BAL fluid. The level was significantly increased in former smokers with emphysema compared with that in former and current smokers without emphysema. Median values are shown as horizontal lines.

reverse transcriptase (Epicentre Technologies, Madison, WI, USA). Briefly, $20 \mu \mathrm{l}$ of reaction mixture contained 10 units of MMLV reverse transcriptase, enzyme buffer, 250 ng total RNA, 20 units RNAse inhibitor (Toyobo, Osaka, Japan), 10 pmol random primer (Takara, Kyoto, Japan), and $1 \mathrm{mM}$ deoxynucleoside triphosphate (Takara). The reaction mixture was incubated at $37^{\circ} \mathrm{C}$ for 60 minutes and chilled on ice.

The $5^{\prime}$ exonuclease based fluorogenic PCR was performed using an ABI PRISM 7700 Sequence Detector (PE Applied Biosystems, Foster City, CA, USA). ${ }^{9}$ Oligonucleotide IL-8 primers and probe for this quantification system were designed to meet specific criteria by using Primer Express software (PE Applied Biosystems) from the published complementary DNA sequences (CDNA) of human IL- $8^{10}$ as follows:

sense: 5'GCAGAGCACACAAGCTTCTAGGA3'

antisense: 5'CCAGCTTGGAAGTCATGTTTACAC3'

probe: 5'CAGGAAGAAACCACCGGAAGGAACCA3'

The predicted size of the amplified IL-8 DNA product was $90 \mathrm{bp}$ and the specific amplification was confirmed by ethidium bromide staining of the PCR product on an agarose gel. The probe was labelled with 6-carboxy-fluorescein at the $5^{\prime}$ end and 6-carboxy-tetramethyl rhodamine at the 3' end (PE Applied Biosystems). Primers and a labelled probe for 

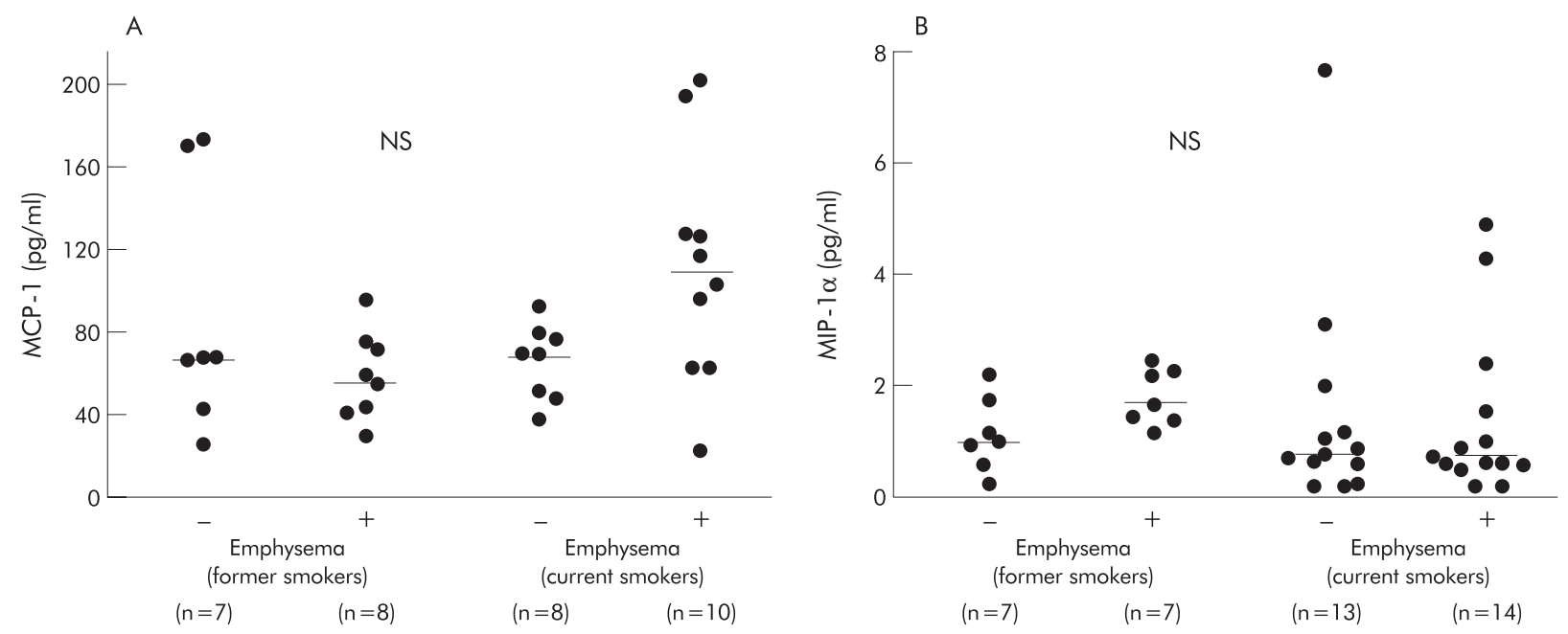

Figure 5 Levels of (A) MCP-1 and (B) MIP-1 $\alpha$ in BAL fluid. There was no significant difference between the four groups. Median values are shown as horizontal lines.
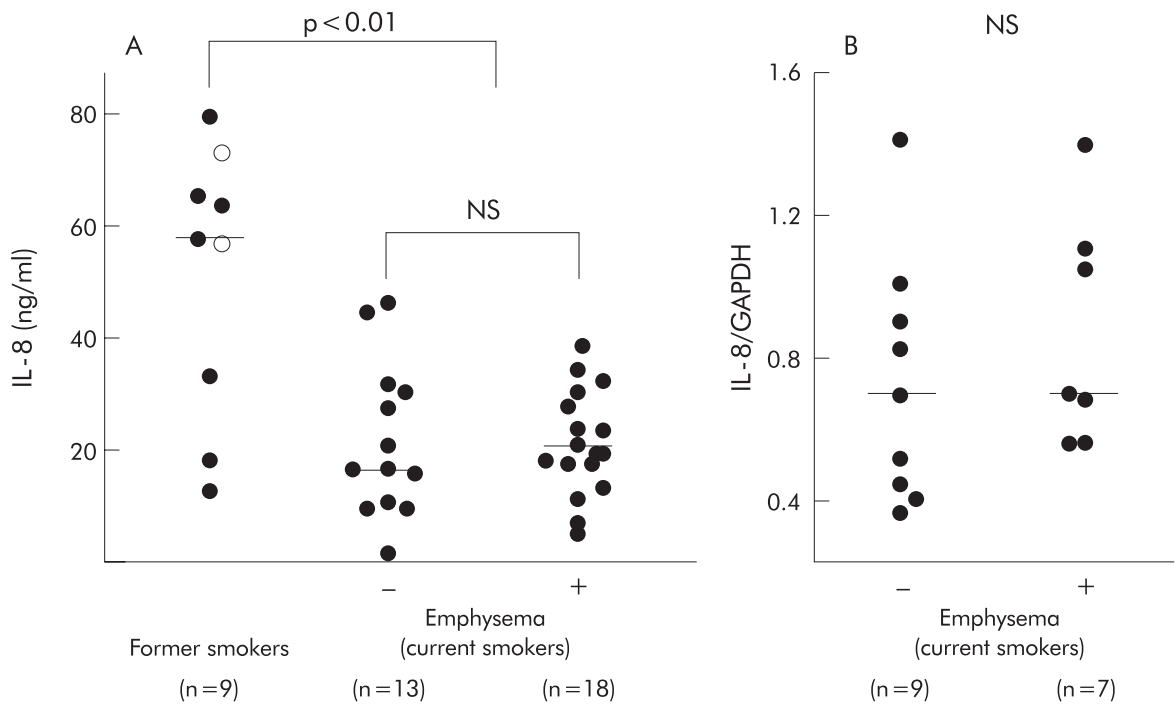

Figure 6 (A) Level of IL-8 in the cultured medium of alveolar macrophages at 24 hours. The level in cultured medium from current smokers was lower than that of the former smokers as a whole $(O=$ former smokers without emphysema $(n=2))$. There was no difference in the level of IL-8 between the two groups of current smokers. Median values are shown as horizontal lines. (B) Level of IL-8 mRNA of alveolar macrophages. Data were normalised by mRNA of GAPDH. There was no significant difference between the two groups of current smokers. Median values are shown as horizontal lines.

glyceraldehyde-3-phosphatase-dehydrogenase (GAPDH) cDNA as an endogenous control were purchased from PE Applied Biosystems (TaqMan GAPDH control reagents). A $5 \mu \mathrm{l}$ aliquot of the RT product was placed into each tube and PCR was carried out in duplicate using a PCR reagent kit (TaqMan PCR core reagents kit with AmpliTaq Gold, PE Applied Biosystems) according to the manufacturer's recommendation (40 cycles; each cycle consisted of a 15 second denaturation step at $95^{\circ} \mathrm{C}$ and a 2 minute combined annealing and extension step at $58^{\circ} \mathrm{C}$ ). IL-8 and GAPDH amplification were performed separately. With this approach, the accurate detection of low concentrations of target mRNA without interference from internal control fluorescence was possible. ${ }^{9}$ The threshold cycle for each sample was determined by the default algorithm of real time quantitative PCR system software (Sequence Detection System, PE Applied Biosystems). Reverse transcribed cDNA of AMs from one subject was serially diluted and used for the generation of the standard curve to assure reproducibility of the quantitative RT-PCR. The relative amount of IL-8 mRNA in the samples was assessed by interpolation of their threshold cycles from a standard curve and then normalised by GAPDH mRNA. This normalised value was used to compare samples.

\section{Statistical analysis}

The demographic data and inflammatory cell profile in BAL fluid were normally distributed and are expressed as mean (SE) values, and the statistical analysis was done by single factor analysis of variance (ANOVA) and Fisher's test for multiple comparisons. As the chemoattractant data were not normally distributed, they were expressed as median values with absolute ranges. Analyses were done with the non-parametric Kruskal-Wallis test and the Mann-Whitney U test. Spearman's rank correlation was calculated to assess the correlation between the data. In all tests values of $<0.05$ were considered statistically significant.

\section{RESULTS}

\section{Characteristics of the subjects}

The 61 subjects were classified into four groups according to their smoking status and the presence or absence of 
emphysema. Emphysema detected on HRCT scans occurred in less than $25 \%$ of the total area of the lungs in most subjects who were categorised as having emphysema. The clinical characteristics and pulmonary function data are shown in table 1. Current smokers were significantly younger than former smokers without emphysema. The smoking index was significantly less in former smokers without emphysema than in subjects in the other three groups. Pulmonary function data revealed that \%VC, $\% \mathrm{FEV}_{1}$, and \%TLco were significantly lower in current smokers and former smokers with emphysema than in former smokers without emphysema. In the current smokers, $\% \mathrm{FEV}_{1}, \mathrm{FEV}_{1} / \mathrm{FVC}$, and \%TLCo were slightly but significantly lower in subjects with emphysema than in those without emphysema.

\section{Inflammatory cell profile in BAL fluid}

The profile of lung inflammatory cells in BAL fluid is shown in table 2 . The recovery rate of BAL fluid was lower in former smokers with emphysema than in current and former smokers without emphysema. When the two groups of current smokers and the two groups of former smokers were combined, current smokers had a higher total cell count and a higher number of AMs than the combined data of former smokers. However, there were no significant differences in the numbers of total cells, macrophages, or neutrophils between the two groups of current smokers classified by the presence or absence of emphysema.

\section{IL-8 in BAL fluid}

IL-8 was detected in the 15-21-fold concentrated BAL fluid of all 54 subjects tested. The level of IL-8 in BAL fluid was significantly increased in current smokers with emphysema (median $34.7 \mathrm{pg} / \mathrm{ml}$; range 5.4-141.3) compared with current smokers without emphysema (median $12.2 \mathrm{pg} / \mathrm{ml}$; range 2.0 62.7; $\mathrm{p}<0.01$ ). The IL-8 level in the latter group was also significantly lower than in former smokers without emphysema (median 25.7 pg/ml; range 9.4-72.7; p<0.05; fig 1). The level of IL-8 was significantly correlated with that of NE- $\alpha$ IPI in BAL fluid ( $\mathrm{n}=54, r=0.65, \mathrm{p}<0.01$; fig 2 ), although no correlation was found either with the absolute number $(r=0.18$, NS) or the percentage ( $r=0.01$, NS) of neutrophils in BAL fluid.

\section{ENA-78 and LTB $_{4}$ in BAL fluid}

ENA-78 was detected in the unconcentrated BAL fluid of 53 subjects tested, with no significant difference between the four groups ( fig 3). $\mathrm{LTB}_{4}$ was detected in the unconcentrated BAL fluid in 50 of 53 subjects tested. The values below the detection limit were assumed to be $3 \mathrm{pg} / \mathrm{ml}$ in the unconcentrated BAL fluid for later statistical analysis. The level was significantly increased in former smokers with emphysema (median $12.4 \mathrm{pg} / \mathrm{ml}$; range 6.0-29.1) but not in current smokers with emphysema (median $9.1 \mathrm{pg} / \mathrm{ml}$; range 3.0-31.7) compared with the two non-emphysematous groups (former smokers: median $7.1 \mathrm{pg} / \mathrm{ml}$; range 3.0-9.3, current smokers: median $9.9 \mathrm{pg} / \mathrm{ml}$; range 3.0-17.3; $<<0.05$; fig 4 ). In contrast with IL-8, there was no significant difference in the levels of ENA-78 and $\mathrm{LTB}_{4}$ between the two groups of current smokers classified by the presence or absence of emphysema.

\section{MCP-1 and MIP- $1 \alpha$ in BAL fluid}

MCP-1 was detected in the concentrated BAL fluid of all 33 subjects tested, and MIP- $1 \alpha$ was detected in concentrated BAL fluid of 35 out of 41 subjects. The values below the detection limit were assumed to be $4 \mathrm{pg} / \mathrm{ml}$ in the concentrated BAL fluid for later statistical analysis. The levels of these chemoattractants were not significantly different between the four groups (fig 5).

\section{IL-8 released from alveolar macrophages}

The cultured media of AMs at 24 hours were diluted 30-fold for the measurement of IL-8. Current smokers had signifi- cantly lower levels of IL-8 than former smokers as a whole (median $17.9 \mathrm{ng} / \mathrm{ml}$ (range 0.3-46.6) v $58.4 \mathrm{ng} / \mathrm{ml}$ (range $12.6-80.8), \mathrm{p}<0.01)$. There was no significant difference in the level of IL- 8 between the two groups of current smokers (fig $6 \mathrm{~A})$.

\section{IL-8 mRNA in alveolar macrophages}

The level of IL-8 mRNA in AMs was quantified using the 5' nuclease fluorogenic RT-PCR. The magnitude of IL-8 mRNA expression corrected by GAPDH transcripts did not differ according to the presence of emphysema in current smokers (fig 6B).

\section{DISCUSSION}

In this study we first demonstrated that IL-8 in BAL fluid could distinguish between current smokers with subclinical emphysema and age matched current smokers without emphysema. In addition, the level of IL-8 in BAL fluid was significantly correlated with that of NE- $\alpha_{1}$ PI. Such an increase in the level of IL-8 in BAL fluid in this group was not associated with an appreciable increase in the number of neutrophils in BAL fluid compared with smoking controls. The level of ENA-78 in BAL fluid was not influenced either by smoking status or by the presence of emphysema. In contrast, the level of $\mathrm{LTB}_{4}$ in BAL fluid was significantly higher in former smokers with emphysema than in the two groups of subjects without emphysema, regardless of smoking status. These data suggest that, although IL-8, ENA-78, and $\mathrm{LTB}_{4}$ are all thought to be primarily chemotactic for neutrophils, their behaviour in the lungs is quite different. On the other hand, the levels of MCP-1 and MIP- $1 \alpha$ in BAL fluid, both of which act mainly on monocytes/macrophages, were not different between the four groups classified by current smoking status and the presence or absence of emphysema. The hypothesis that AMs are one of the sources of IL-8 contributing to the early development of pulmonary emphysema was found to be unlikely because both spontaneous release of IL- 8 at 24 hours in the culture medium of AMs and the expression of IL-8 mRNA in AMs were similar in the two groups of current smokers with and without emphysema.

IL-8 is a potent chemoattractant that is needed to recruit and activate neutrophils. ${ }^{5}$ It maintains its biological activity in the presence of significant changes in $\mathrm{pH}$ and resists mild proteolytic degradation compared with other known chemoattractants. ${ }^{11}$ As a result, it may prolong the activity of recruited neutrophils at sites of inflammation. We have shown that current smokers with subclinical emphysema do not have an increased number of neutrophils in BAL fluid compared with current smokers with a comparable smoking history without any signs of emphysema based on HRCT scans. We have, however, provided evidence of neutrophil degranulation in the lungs of these subjects because they have increased levels of neutrophil granule proteins in BAL fluid such as NE- $\alpha_{1} \mathrm{PI}, \mathrm{HNL}$, MMP-8, and MMP-9. Although the source of MMP-9 is not thought to be neutrophils alone, we showed in a previous study that some fractions of MMP-9 detected in BAL fluid from subjects with subclinical emphysema form a complex with HNL, indicating the neutrophil origin of MMP-9. ${ }^{4}$

Several previous studies have shown that patients with clinically apparent chronic obstructive pulmonary disease (COPD), who are thought to have neutrophil inflammation of airways, have increased levels of IL-8 in induced sputum or BAL fluid. ${ }^{12}{ }^{13}$

On the other hand, there has been controversy about whether the level of IL-8 in the lungs of healthy smokers is increased ${ }^{13}$ or not. ${ }^{14}$ Our study population differs from those in previous studies because we separated subjects with subclinical emphysema evident on HRCT scans from healthy smokers. These characteristics indicate that the subjects are in the early stage of pulmonary emphysema. We thus speculate that 
smokers with an increased level of IL-8 in previous studies might be those who were developing or would later develop pulmonary emphysema.

ENA-78 was first identified from conditioned medium of stimulated human type II epithelial cell line A549 which, like IL-8, belongs to the $\mathrm{C}-\mathrm{X}-\mathrm{C}$ chemokine superfamily. ${ }^{5}$ In acute respiratory distress syndrome (ARDS) the levels of both cytokines were similarly increased in BAL fluid and had a good correlation with the number of neutrophils. ${ }^{15} \mathrm{LTB}_{4}$ is one of the lipoxygenase products of arachidonic acid. ${ }^{7}$ In COPD the levels of IL-8 and $\mathrm{LTB}_{4}$ were similarly increased in sputum and had positive correlations with neutrophil elastase activity. ${ }^{16}$ Like IL-8, both ENA-78 and $\mathrm{LTB}_{4}$ are potent chemoattractants as well as activators for neutrophils. Generally, all three of these chemoattractants were found in high concentrations under inflammatory conditions where neutrophils were thought to be heavily involved. However, they differ in the pattern of expression in response to exogenous stimuli in vitro, although they are produced and released from cultured monocytes and/or macrophages. ${ }^{17}{ }^{18}$ In this study we have shown that these chemoattractants may play different roles, at least in the early developing stage of pulmonary emphysema.

The likely explanation for the dissociation between the number of neutrophils and the level of NE- $\alpha_{1}$ PI or IL-8 in BAL fluid may be that the neutrophils harvested by BAL do not necessarily reflect the total number of neutrophils in the lung. Neutrophils stay not only in the air spaces but also in the interstitium or in the pulmonary microvessels. In addition, some neutrophils may be firmly adherent to the airways and are not washed out of the lungs. An alternative explanation is that IL- 8 is released by the more active neutrophils, and this accounts for the correlation with NE- $\alpha_{1}$ PI. Finally, IL- 8 is not present in sufficient quantities to lead to significant neutrophil recruitment, but does activate the neutrophils in subjects with subclinical emphysema. A hypothesis that circulating activated neutrophils were implicated in the pathogenesis of COPD was first proposed by Burnett and others. ${ }^{19}$

It is worth noting that there were no significant differences in the levels of MCP-1 or of MIP- $1 \alpha$ in BAL fluid from the four groups of subjects, although the number of AMs was increased in both groups of current smokers to a similar extent to that in the groups of former smokers. One earlier study showed that the BAL fluid of smokers had a significantly increased capacity to attract blood monocytes and that the chemokinetic activity correlated with the number of AMs in the BAL fluid. However, the authors of that study did not identify any specific chemoattractants..$^{20}$ We thus speculate that there are chemoattractants other than MCP-1 or MIP- $1 \alpha$ which could account for the increase in the number of AMs in BAL fluid from current smokers. On the other hand, a few recent studies have shown an important role for $\mathrm{MCP}-\mathrm{I}^{21}$ and/or MIP- $1 \alpha^{22}$ in the accumulation of macrophages in the lungs of patients with COPD. However, the present study does not support the idea that these chemoattractants could be factors that distinguish smokers with emphysema from those without emphysema, at least in the early stage of the disease.

Among the subjects with emphysema there were no relationships between the levels of inflammatory chemoattractants in BAL fluid and the degree of emphysema on CT scans or their pulmonary function. However, it should be noted that patients with clinically apparent emphysema were not included in our study and almost all the subjects who were classified as having emphysema had low attenuation areas comprising less than $25 \%$ of the total area, if any, on CT scans. Accordingly, it is hard to discuss the relationship between disease severity and the levels of inflammatory chemoattractants measured. A possible explanation for the variable levels of IL-8 and other proteins among the subjects with emphysema is the heterogeneity of emphysematous changes in the early stage in individual lungs. The fluid lavaged from the middle lobe of the lung may not necessarily reflect the phenomena at the site of emphysema.
As the level of IL-8 was increased in BAL fluid from smokers with subclinical emphysema, we anticipated that the release of IL-8 from AMs would be accelerated in these subjects, as is the case in patients with idiopathic pulmonary fibrosis. ${ }^{23}$ We were unable to attribute the increase in IL-8 levels in BAL fluid to AMs; neither the release of IL-8 from cultured AMs at 24 hours nor the expression of IL-8 mRNA of AMs was increased at all in those subjects compared with the smoking controls. However, we should interpret these negative results with caution. Because macrophages are studied in culture ex vivo, any stimulating agent that could have overactivated the macrophages while in the patient would now be absent, and might also account for a negative result. In addition, the release of IL-8 from AMs was suppressed in current smokers compared with former smokers when the subjects were combined, regardless of the presence of emphysema. A similar suppression by chronic smoking of chemoattractant production from AMs has been shown both in vitro and in vivo for $\mathrm{LTB}_{4}$, TNF- $\alpha$, and others. ${ }^{24} 25$

It is known that IL-8 is produced and released by a variety of cells in the lungs, including neutrophils, T lymphocytes, fibroblasts, and epithelial cells. ${ }^{5}$ Airway epithelial cells are considered to be a particularly important source of this chemoattractant. ${ }^{26} \mathrm{~A}$ recent study demonstrated by immunohistochemistry and in situ hybridisation that IL-8 in bronchiolar and alveolar epithelium is more intensely positive in smokers with COPD than in smokers without COPD. ${ }^{21}$ Furthermore, cigarette smoke is reported to enhance IL-8 release from bronchial epithelial cells. ${ }^{27}$ Taken together with our present data, it is tempting to speculate that intersubject variation in the IL- 8 response to cigarette smoke of bronchial and/or alveolar epithelial cells is a determinant for the early development of pulmonary emphysema.

Finally, it is possible that the inflammatory pattern in the lungs of patients with COPD may differ according to the phenotype and/or the clinical stage of the disease. Numerous studies exist that place emphasis on the roles of macrophages, mast cells, CD8+ lymphocytes, and eosinophils in the development and progression of COPD. ${ }^{28}{ }^{29}$ Our previous study also suggested a diverse contribution of neutrophil elastase and cathepsin L to the development of subclinical emphysema in populations of various ages. ${ }^{30}$ We therefore feel that the importance of neutrophil activation and the specific role of IL-8 found in our series of studies may be true only in the early stage of pulmonary emphysema.

In summary, we have shown that the level of IL-8, but not of ENA-78 or $\mathrm{LTB}_{4}$, in BAL fluid from current smokers with subclinical emphysema is significantly increased compared with age matched current smokers without emphysema. Taken together with our previous studies which found increased levels of neutrophil granule proteins in BAL fluid from subjects with subclinical emphysema, these data suggest that IL- 8 may be a key factor that distinguishes smokers who are developing emphysema from those who are not, at least in the early stage of the disease. Since AMs were not likely to be the source of IL-8 contributing to subclinical emphysema in this study, further studies should focus on the airway epithelial function as a possible candidate for intersubject variation in the susceptibility to smoking induced lung destruction.

\section{ACKNOWLEDGEMENTS}

The authors thank Dr Yoshikazu Kawakami (Konan Hospital, Sapporo, Japan) for his constructive comments and Dr Natsuko Kitashiro (Nanae Hospital, Nanae, Japan) for her advice regarding the RT-PCR technique.

\section{Authors' affiliations}

M Tanino, T Betsuyaku, K Takeyabu, Y Tanino, E Yamaguchi, K Miyamoto, M Nishimura, First Department of Medicine, Hokkaido University School of Medicine, Kita-ku, Sapporo, 060-8638, Japan

No part of the research presented has been funded by tobacco industry sources. 


\section{REFERENCES}

1 Senior RM, Shapiro SD. Chronic obstructive pulmonary disease: epidemiology, pathophysiology, and pathogenesis. In: Fishman's pulmonary diseases and disorders. 3rd ed. New York: McGraw-Hill, 1997: 659-81.

2 Yoshioka A, Betsuyaku T, Nishimura M, et al. Excessive neutrophil elastase in bronchoalveolar lavage fluid in subclinical emphysema. Am J Respir Crit Care Med 1995;152:2127-32.

3 Betsuyaku T, Nishimura M, Yoshioka A, et al. Elastin-derived peptides and neutrophil elastase in bronchoalveolar lavage fluid. Am J Respir Crit Care Med 1996;154:720-4.

4 Betsuyaku T, Nishimura M, Takeyabu K, et al. Neutrophil granule proteins in bronchoalveolar lavage fluid from subjects with subclinical emphysema. Am J Respir Crit Care Med 1999;159:1985-91.

5 Baggiolini M, Dewald B, Moser B. Interleukin-8 and related chemotactic cytokines: CXC and CC chemokines. Adv Immunol 1994;55:97-179.

6 Walz A, Burgener R, Car B, et al. Structure and neutrophil-activating properties of a novel inflammatory peptide (ENA-78) with homology to interleukin 8. J Exp Med 1991;174:1355-62

7 Lewis RA, Austen KF. The biologically active leukotrienes. Biosynthesis, metabolism, receptors, functions, and pharmacology. J Clin Invest 1984;73:889-97.

8 Betsuyaku T, Yoshioka A, Nishimura M, et al. Neutrophil elastase associated with alveolar macrophages from older volunteers. Am J Respir Crit Care Med 1995;151:436-42

9 Gibson UEM, Heid CA, Williams PM. A novel method for real time quantitative RT-PCR. Genome Res 1996;6:995-1001.

10 Matsushima K, Morishita K, Yoshimura T, et al. Molecular cloning of a human monocyte-derived neutrophil chemotactic factor (MDNCF) and the induction of MDNCF mRNA by interleukin 1 and tumor necrosis factor. $J$ Exp Med 1988;167:1883-93

11 Steieter RM, Lukacs NW, Standiford TJ, et al. Cytokines and lung inflamation: mechanisms of neutrophil recruitment to the lung. Thorax 1993;48:765-9.

12 Richman-Eisenstat JB, Jorens PG, Hebert CA, et al. Interleukin-8: an important chemoattractant in sputum of patients with chronic inflammatory airway diseases. Am J Physiol 1993;264:L413-8.

13 Keatings VM, Collins PD, Scott DM, et al. Differences in interleukin-8 and tumor necrosis factor- $\alpha$ in induced sputum from patients with chronic obstructive pulmonary disease or asthma. Am J Respir Crit Care Med 1996;153:530-4.

14 Morrison D, Strieter RM, Donnelly SC, et al. Neutrophil chemokines in bronchoalveolar lavage fluid and leukocyte-conditioned medium from nonsmokers and smokers. Eur Respir J 1998;12:1067-72.

15 Goodman RB, Strieter RM, Martin DP, et al. Inflammatory cytokines in patients with persistence of the acute respiratory distress syndrome. Am Respir Crit Care Med 1996;154:602-11.
16 Hill AT, Bayley D, Stockley RA. The interrelationship of sputum inflammatory markers in patients with chronic bronchitis. Am J Respir Crit Care Med 1999; 160:893-8

17 Schnyder-Candrian S, Walz A. Neutrophil-activating protein ENA-78 and IL-8 exhibit different patterns of expression in lipopolysaccharideand cytokine-stimulated human monocyte. J Immunol 1997;158:388894.

18 Rankin JA, Sylvester I, Smith S, et al. Macrophages cultured in vitro release leukotriene B4 and neutrophil attractant/ activation protein (interleukin 8) sequentially in response to stimulation with lipopolysaccharide and zymosan. J Clin Invest 1990;86:1556-64.

19 Burnett D, Chamba A, Hill SL, et al. Neutrophils from subjects with chronic obstructive lung disease show enchanced chemotaxis and extracellular proteolysis. Lancet 1987;1043-6.

20 Koyama S, Rennard SI, Daughton D, et al. Bronchoalveolar lavage fluid obtained from smokers exhibits increased monocyte chemokinetic activity. J Appl Physiol 1991;70:1208-14.

21 De Boer WI, Sont JK, van Schadewijk A, et al. Monocyte chemoattractant protein 1 , interleukin 8 , and chronic airways inflammation in COPD. J Pathol 2000;190:619-26.

22 Di Stefano A, Capelli A, Lusuardi M, et al. Severity of airflow limitation is associated with severity of airway inflammation in smokers. Am J Respir Crit Care Med 1998;158:1277-85.

23 Nakamura $\mathbf{H}$, Fujishima S, Waki Y, et al. Priming of alveolar macrophages for interleukin-8 production in patients with ideopathic pulmonary fibrosis. Am J Respir Crit Care Med 1995;152:1579-86.

24 Laviolette, M, Coulombe R, Picard S, et al. Decreased leukotriene B4 synthesis in smokers' alveolar macrophages in vitro. J Clin Invest 1986;77:54-60.

25 Yamaguchi $\mathbf{E}$, Itoh A, Furuya K, et al. Release of tumor necrosis factor- $\alpha$ from human alveolar macrophages is decreased in smokers. Chest 1993; 103:479-83.

26 Takizawa H, Tanaka M, Takami K, et al. Increased expression of inflammatory mediators in small-airway epithelium from tobacco smokers. Am J Physiol 2000;278:L906-13

27 Mio T, Romberger DJ, Thompson AB, et al. Cigarette smoke induces interleukin-8 release from human bronchial epithelial cells. Am J Respir Crit Care Med 1997;155:1770-6.

28 Cosio MG, Guerassimov A. Chronic obstructive pulmonary disease: inflammation of small airways and lung parenchyma. Am J Respir Crit Care Med 1999;160:S21-5

29 Grashoff WFH, Sont JK, Sterk PJ, et al. Chronic obstructive pulmonary disease: role of bronchiolar mast cells and macrophages. Am J Pathol 1997; 151:1785-90.

30 Takeyabu K, Betsuyaku T, Nishimura M, et al. Cysteine proteases and cystatin $\mathrm{C}$ in bronchoalveolar lavage fluid from subjects with subclinical emphysema. Eur Respir J 1998;12:1033-9. 